\title{
Second-Order Discontinuities for Simple and Double Layers
}

\author{
M. Grinfeld ${ }^{1,2}$, P. Grinfeld 1,2 \\ ${ }^{1}$ U.S. Army Laboratory, Aberdeen Proving Ground, MD, USA \\ ${ }^{2}$ Drexel University, Philadelphia, PA, USA \\ Email: michael.greenfield4.civ@mail.mil
}

How to cite this paper: Grinfeld, M. and Grinfeld, P. (2021) Second-Order Discontinuities for Simple and Double Layers. Journal of Applied Mathematics and Physics, 9, 2205-2214.

https://doi.org/10.4236/jamp.2021.99140

Received: June 9, 2021

Accepted: September 11, 2021

Published: September 14, 2021

Copyright $\odot 2021$ by author(s) and Scientific Research Publishing Inc. This work is licensed under the Creative Commons Attribution International License (CC BY 4.0).

http://creativecommons.org/licenses/by/4.0/

\begin{abstract}
Simple and double layers first appeared in electrostatics and later found various applications in mathematical physics. In this paper, we present the jump discontinuity conditions for their second-order spatial derivatives.
\end{abstract}

\section{Keywords}

Simple and Double Layers, Discontinuous Solutions, Electromagnetics

\section{Introduction}

In his 1887 paper [1], Weingarten announced an elegant formula, found in Equation (58) below, for the jump discontinuities in the second derivatives of the Newtonian potential across the boundary of a self-gravitating body. Recall that the Newtonian potential $V_{\rho}$ induced by the mass density distribution $\rho$ over a domain $\Omega^{+}$is given by

$$
V_{\rho}(Z)=-\int_{\Omega^{+}} \frac{\rho(Z)}{\left|\vec{Z}(Z)-\vec{Z}\left(Z^{*}\right)\right|} d Z^{*},
$$

where $\vec{Z}$ is the radius vector and the symbols $V(Z)$ and $\vec{Z}(Z)$ denote the functions $V\left(Z^{1}, Z^{2}, Z^{3}\right)$ and $\vec{Z}\left(Z^{1}, Z^{2}, Z^{3}\right)$ of the coordinates $Z^{i}$.

Our goal is to construct formulas analogous to Equation (58) for the jump discontinuities in the potentials induced by simple and double layer distributions over a surface $S$. A simple layer potential $V_{\sigma}$ is usually associated with a charge distribution $\sigma(S)$ while a double layer potential $V_{\delta}$ is usually associated with a dipole distribution $\delta(S)$. They are given by the surface integrals

$$
V_{\sigma}(Z)=\int_{S} \frac{\mu(S)}{|\vec{Z}(Z)-\vec{Z}(S)|} \mathrm{d} S
$$


and

$$
V_{\delta}(Z)=-\int_{S} \delta(S) \frac{\partial}{\partial n} \frac{1}{|\vec{Z}(Z)-\vec{Z}(S)|} \mathrm{d} S
$$

where $\partial / \partial n$ denotes the normal derivative. The relevant elements of potential theory can be found in the classic texts [2]-[7].

\section{The Relevant Elements of Tensor Calculus}

Let us briefly summarize our notation, as well as the basic elements of tensor calculus that we will use in this paper. Refer the three-dimensional Euclidean space to general curvilinear coordinates $Z^{i}$. The Latin indices will run the values 1,2 , and 3. Treat the radius vector $\vec{Z}$ as a function of the coordinates denoted by $\vec{Z}\left(Z^{1}, Z^{2}, Z^{3}\right)$. We will typically collapse the arguments of functions and denote $\vec{Z}\left(Z^{1}, Z^{2}, Z^{3}\right)$ simply by $\vec{Z}(Z)$. Let

$$
\vec{Z}_{i}=\frac{\partial \vec{Z}(Z)}{\partial Z^{i}}
$$

be the covariant basis and

$$
Z_{i j}=\vec{Z}_{i} \cdot \vec{Z}_{j}
$$

be the covariant metric tensor. The contravariant metric tensor $Z^{i j}$ is defined as the matrix inverse of $Z_{i j}$. We will use the standard elements of tensor calculus in the Euclidean space including the multiplication and contraction of tensors, index juggling, and covariant differentiation. For the necessary background in tensor calculus, we especially recommend [8] as well as the book [9] by Tracy Thomas who was one of the key figures in the theory of compatibility conditions for discontinuous tensorial fields. We also recommend our books [10] [11] from which we borrow the notation and some of the elementary results with regard to the compatibility conditions.

Now consider a surface $S$. Let the Greek indices run the values 1 and 2 and refer the our surface $S$ to the coordinate $S^{\alpha}$. Suppose that

$$
Z^{i}=Z^{i}(S)
$$

are the equations of the surface $S$, where the symbol $Z^{i}(S)$ represents the functions $Z^{i}\left(S^{1}, S^{1}\right)$. Let $\vec{Z}(S)$ be the surface restriction of the ambient radius vector function $\vec{Z}(Z)$. Then $\vec{Z}(S)$ is given by the composition

$$
\vec{Z}(S) \equiv \vec{Z}(Z(S)) \text {. }
$$

The shift tensor $Z_{\alpha}^{i}$ is given by

$$
Z_{\alpha}^{i}(S) \equiv \frac{\partial Z^{i}(S)}{\partial S^{\alpha}} .
$$

The surface covariant basis, given by

$$
\vec{S}_{\alpha}=\frac{\partial \vec{Z}(S)}{\partial S^{\alpha}},
$$


is related to the ambient covariant basis $\vec{Z}_{i}$ by the identity

$$
\vec{S}_{\alpha}=\vec{Z}_{i} Z_{\alpha}^{i} \text {. }
$$

The surface covariant metric tensor is given by

$$
S_{\alpha \beta} \equiv \vec{S}_{\alpha} \cdot \vec{S}_{\beta} .
$$

Its matrix inverse is the contravariant metric tensor $S^{\alpha \beta}$. All objects on the surface are subject to the surface covariant derivative denoted by $\nabla_{\alpha}$.

The shape of the boundary $S$ is characterized by the unit normal $N^{i}$ and the curvature tensor $B_{\alpha \beta}$, also known as the second fundamental form. These important tensors satisfy the equations

$$
\begin{gathered}
Z_{i}^{\alpha} Z_{\beta}^{i}=\delta_{\beta}^{\alpha} \\
N_{i} N^{i}=1 \\
N_{i} Z_{\alpha}^{i}=0 \\
\nabla_{\beta} Z_{\alpha}^{i}=B_{\alpha \beta} N^{i} \\
\nabla_{\beta} N^{i}=-B_{\beta}^{\alpha} Z_{\alpha}^{i},
\end{gathered}
$$

where the last equation is known as Weingarten's equation. Finally, we will make frequent use of the projection formula

$$
\delta_{j}^{i}=N^{i} N_{j}+Z_{\alpha}^{i} Z_{j}^{\alpha},
$$

although it will almost always appear with different combinations of index names.

\section{One-Sided Limits and Jump Discontinuities}

Consider a field $F(Z)$ defined in the ambient space surrounding the interface $S$ referred to surface coordinates $S^{\alpha}$. Suppose that $S$ divides the space into two regions denoted by $\Omega^{+}$and $\Omega^{-}$. Consider a point $P$ on $S$ with ambient coordinates $Z_{0}^{i}$ and surface coordinates $S^{\alpha}$. Then let $F^{+}(S)$ be the limiting value of $F(Z)$ as $Z^{i} \rightarrow Z^{i}(S)$ from the region $\Omega^{+}$and, similarly, $F^{-}(S)$ be the limiting value of $F(Z)$ as $Z^{i} \rightarrow Z^{i}(S)$ from the region $\Omega^{-}$. The fields $F^{+}(S)$ and $F^{-}(S)$ defined on the surface $S$ are known as one-sided limits of $F(Z)$ and are illustrated in Figure 1.

The same concepts can be applied to tensor fields such as $\nabla_{i} F$, where the symbols $\nabla_{i} F^{+}$and $\nabla_{i} F^{-}$are understood as the one-sided limits of $\nabla_{i} F$.

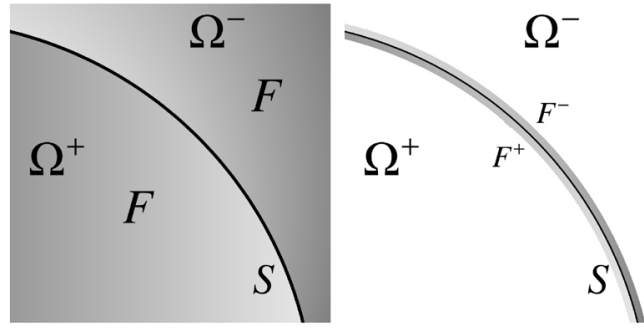

Figure 1. Ilustration of the concept of a one-side limit. 
(Note that the ambient derivative $\nabla_{i}$ cannot be applied to $F^{+}$and $F^{-}$since the latter are defined only on the surface.)

Recall that the surface covariant derivative $\nabla_{\alpha}$ satisfies the chain rule

$$
\nabla_{\alpha}=Z_{\alpha}^{i} \nabla_{i}
$$

when applied to fields defined in the surrounding ambient space. Therefore,

$$
\nabla_{\alpha} F^{+}=Z_{\alpha}^{i} \nabla_{i} F^{+}
$$

and

$$
\nabla_{\alpha} F^{-}=Z_{\alpha}^{i} \nabla_{i} F^{-},
$$

assuming that the covariant derivative and the limit can be interchanged. This is known as the Hadamard lemma. A rigorous derivation of this lemma can be found in [7].

For a field $F$ that is discontinuous across $S$, the quantity $F^{+}-F^{-}$is called the jump discontinuity and, according to notation introduced above, is denoted by $[F]$, i.e.

$$
[F]=F^{+}-F^{-} \text {. }
$$

From Equations (19) and (20), we find that

$$
\nabla_{\alpha}[F]=Z_{\alpha}^{i}\left[\nabla_{i} F\right] .
$$

Contract both sides of this equation with $Z_{j}^{\alpha}$, i.e.

$$
Z_{j}^{\alpha} \nabla_{\alpha}[F]=Z_{j}^{\alpha} Z_{\alpha}^{i}\left[\nabla_{i} F\right] \text {. }
$$

Since (see [8] and [9])

$$
Z_{j}^{\alpha} Z_{\alpha}^{i}=\delta_{j}^{i}-N^{i} N_{j}
$$

we have

$$
Z_{j}^{\alpha} \nabla_{\alpha}[F]=\left[\nabla_{j} F\right]-N_{j} N^{i}\left[\nabla_{i} F\right] .
$$

Exchanging the names of the indices $i$ and $j$ and solving for $\left[\nabla_{i} F\right]$, we find

$$
\left[\nabla_{i} F\right]=Z_{i}^{\alpha} \nabla_{\alpha}[F]+N_{i} N^{j}\left[\nabla_{j} F\right] \text {. }
$$

This formula "decomposes" the jump discontinuity $\left[\nabla_{i} F\right]$ of the derivatives $\nabla_{i} F$ in terms of the jump discontinuity $[F]$ of $F$ itself and the jump discontinuity $N^{j}\left[\nabla_{j} F\right]$ of the normal components of $\nabla_{i} F$. The latter can be thought of as the jump discontinuity in the normal derivative $\partial F / \partial n$ of $F$.

Let $H_{n}$ denote the jump discontinuity in the $n$-th normal derivative of $F$. In other words,

$$
\begin{gathered}
H_{0}=[F] \\
H_{1}=N^{k}\left[\nabla_{k} F\right] \\
H_{2}=N^{k} N^{l}\left[\nabla_{k} \nabla_{l} F\right],
\end{gathered}
$$

and so on. With the help of these symbols, Equation (26) can be rewritten as

$$
\left[\nabla_{i} F\right]=Z_{i}^{\alpha} \nabla_{\alpha} H_{0}+N_{i} H_{1} \text {. }
$$


Equation (30) is called the first-order compatibility conditions. The $N^{\text {th }}$-order compatibility conditions are an expression for the jump discontinuities in the $N^{\text {th }}$-order derivatives of $F$ in terms of the $H_{n \leq N}$. In essence, compatibility conditions treat the quantities $H_{n}$ as the degrees of freedom governing the jump discontinuities and thus provide a great deal of structure and insight.

The goal of the next section is to derive the general second-order compatibility conditions which can then be applied to the simple and double layer potentials $V_{\sigma}$ and $V_{\delta}$.

\section{The General Second-Order Compatibility Conditions}

Our present goal is to derive the compatibility conditions for the discontinuity jumps $\left[\nabla_{i} \nabla_{j} F\right]$ in the second derivative of $F$. With the help of the projection formula

$$
\delta_{j}^{k}=N^{k} N_{i}+Z_{\alpha}^{k} Z_{i}^{\alpha},
$$

$\left[\nabla_{i} \nabla_{j} F\right]$ can be rewritten as

$$
\begin{aligned}
{\left[\nabla_{i} \nabla_{j} F\right] } & =\left[\nabla_{k} \nabla_{j} F\right] \delta_{i}^{k} \\
& =\left[\nabla_{k} \nabla_{j} F\right]\left(N^{k} N_{i}+Z_{\alpha}^{k} Z_{i}^{\alpha}\right) \\
& =\left[\nabla_{k} \nabla_{j} F\right] N^{k} N_{i}+Z_{\alpha}^{k} Z_{i}^{\alpha}\left[\nabla_{k} \nabla_{j} F\right] .
\end{aligned}
$$

By the chain rule,

$$
Z_{\alpha}^{k} Z_{i}^{\alpha}\left[\nabla_{k} \nabla_{j} F\right]=Z_{i}^{\alpha} \nabla_{\alpha}\left[\nabla_{j} F\right]
$$

and therefore

$$
\left[\nabla_{i} \nabla_{j} F\right]=\left[\nabla_{k} \nabla_{j} F\right] N^{k} N_{i}+Z_{i}^{\alpha} \nabla_{\alpha}\left[\nabla_{j} F\right] .
$$

We will now analyze each of the terms on the right separately.

\subsection{The Term $\left[\nabla_{k} \nabla_{j} F\right] N^{k} N_{i}$}

Another application of the projection formula

$$
\delta_{j}^{l}=N^{l} N_{j}+Z_{\alpha}^{l} Z_{j}^{\alpha}
$$

yields

$$
\begin{aligned}
{\left[\nabla_{k} \nabla_{j} F\right] N^{k} N_{i} } & =\left[\nabla_{k} \nabla_{l} F\right] \delta_{j}^{l} N^{k} N_{i} \\
& =\left[\nabla_{k} \nabla_{l} F\right]\left(N^{l} N_{j}+Z_{\alpha}^{l} Z_{j}^{\alpha}\right) N^{k} N_{i} \\
& =\left[\nabla_{k} \nabla_{l} F\right] N^{l} N_{j} N^{k} N_{i}+\left[\nabla_{k} \nabla_{l} F\right] Z_{\alpha}^{l} Z_{j}^{\alpha} N^{k} N_{i} \\
& =H_{2} N_{i} N_{j}+Z_{j}^{\alpha} \nabla_{\alpha}\left[\nabla_{k} F\right] N^{k} N_{i}
\end{aligned}
$$

Since

$$
\begin{aligned}
\nabla_{\alpha}\left[\nabla_{k} F\right] N^{k} & =\nabla_{\alpha}\left(N^{k}\left[\nabla_{k} F\right]\right)-\left[\nabla_{k} F\right] \nabla_{\alpha} N^{k} \\
& =\nabla_{\alpha}\left(N^{k}\left[\nabla_{k} F\right]\right)+Z_{\beta}^{k}\left[\nabla_{k} F\right] B_{\alpha}^{\beta} \\
& =\nabla_{\alpha} H_{1}+B_{\alpha}^{\beta} \nabla_{\beta} H_{0},
\end{aligned}
$$


we have

$$
\left[\nabla_{k} \nabla_{j} F\right] N^{k} N_{i}=H_{2} N_{i} N_{j}+N_{i} Z_{j}^{\alpha} \nabla_{\alpha} H_{1}+N_{i} Z_{j}^{\alpha} B_{\alpha}^{\beta} \nabla_{\beta} H_{0} .
$$

\subsection{The Term $Z_{i}^{\alpha} \nabla_{\alpha}\left[\nabla_{j} F\right]$}

Let us once again use the project formula

$$
\delta_{j}^{k}=N^{k} N_{j}+Z_{j}^{\beta} Z_{\beta}^{k} .
$$

We have

$$
\begin{aligned}
Z_{i}^{\alpha} \nabla_{\alpha}\left[\nabla_{j} F\right] & =Z_{i}^{\alpha} \nabla_{\alpha}\left(\left[\nabla_{k} F\right] \delta_{j}^{k}\right) \\
& =Z_{i}^{\alpha} \nabla_{\alpha}\left(\left[\nabla_{k} F\right] N^{k} N_{j}+\left[\nabla_{k} F\right] Z_{j}^{\beta} Z_{\beta}^{k}\right) \\
& =Z_{i}^{\alpha} \nabla_{\alpha}\left(H_{1} N_{j}+Z_{j}^{\beta} \nabla_{\beta} H_{0}\right) .
\end{aligned}
$$

Continuing with the product rule, we have

$$
Z_{i}^{\alpha} \nabla_{\alpha}\left[\nabla_{j} F\right]=Z_{i}^{\alpha}\left(\nabla_{\alpha} Z_{j}^{\beta} \nabla_{\beta} H_{0}+Z_{j}^{\beta} \nabla_{\alpha} \nabla_{\beta} H_{0}\right)+Z_{i}^{\alpha}\left(\nabla_{\alpha} H_{1} N_{j}+H_{1} \nabla_{\alpha} N_{j}\right)
$$

Since $\nabla_{\alpha} Z_{j}^{\beta}=N_{j} B_{\alpha}^{\beta}$ and $\nabla_{\alpha} N_{j}=-Z_{j}^{\beta} B_{\alpha \beta}$, we find

$$
Z_{i}^{\alpha} \nabla_{\alpha}\left[\nabla_{j} F\right]=Z_{i}^{\alpha} Z_{j}^{\beta} \nabla_{\alpha} \nabla_{\beta} H_{0}+Z_{i}^{\alpha} N_{j} B_{\alpha}^{\beta} \nabla_{\beta} H_{0}+N_{j} Z_{i}^{\alpha} \nabla_{\alpha} H_{1}-Z_{i}^{\alpha} Z_{j}^{\beta} B_{\alpha \beta} H_{1} \text {. }
$$

The combination $Z_{i}^{\alpha} Z_{j}^{\beta} B_{\alpha \beta}$ is often denoted by $B_{i j}$, i.e.

$$
B_{i j}=Z_{i}^{\alpha} Z_{j}^{\beta} B_{\alpha \beta} .
$$

With the help of this new symbol, $Z_{i}^{\alpha} \nabla_{\alpha}\left[\nabla_{j} F\right]$ is given by

$$
Z_{i}^{\alpha} \nabla_{\alpha}\left[\nabla_{j} F\right]=Z_{i}^{\alpha} Z_{j}^{\beta} \nabla_{\alpha} \nabla_{\beta} H_{0}+Z_{i}^{\alpha} N_{j} B_{\alpha}^{\beta} \nabla_{\beta} H_{0}+N_{j} Z_{i}^{\alpha} \nabla_{\alpha} H_{1}-B_{i j} H_{1} .
$$

\subsection{The Final Relationship}

Combine the two terms obtained in the preceding sections:

$$
\begin{aligned}
{\left[\nabla_{i} \nabla_{j} F\right]=} & H_{2} N_{i} N_{j}+N_{i} Z_{j}^{\alpha} \nabla_{\alpha} H_{1}+N_{i} Z_{j}^{\alpha} B_{\alpha}^{\beta} \nabla_{\beta} H_{0}+Z_{i}^{\alpha} Z_{j}^{\beta} \nabla_{\alpha} \nabla_{\beta} H_{0} \\
& +Z_{i}^{\alpha} N_{j} B_{\alpha}^{\beta} \nabla_{\beta} H_{0}+N_{j} Z_{i}^{\alpha} \nabla_{\alpha} H_{1}-B_{i j} H_{1} .
\end{aligned}
$$

Collecting like terms we arrive at the final second-order compatibility conditions

$$
\begin{aligned}
{\left[\nabla_{i} \nabla_{j} F\right]=} & H_{2} N_{i} N_{j}+\left(N_{i} Z_{j}^{\alpha}+N_{j} Z_{i}^{\alpha}\right) \nabla_{\alpha} H_{1}-B_{i j} H_{1} \\
& +\left(N_{j} Z_{i}^{\alpha}+N_{i} Z_{j}^{\alpha}\right) B_{\alpha}^{\beta} \nabla_{\beta} H_{0}+Z_{i}^{\alpha} Z_{j}^{\beta} \nabla_{\alpha} \nabla_{\beta} H_{0} .
\end{aligned}
$$

Let us call attention to several important special cases. If the field $F$ is continuous across $S$, i.e. $H_{0}=0$, then

$$
\left[\nabla_{i} \nabla_{j} F\right]=H_{2} N_{i} N_{j}+\left(N_{i} Z_{j}^{\alpha}+N_{j} Z_{i}^{\alpha}\right) \nabla_{\alpha} H_{1}-B_{i j} H_{1} .
$$

If $F$ has continuous first-order derivatives across $S$, i.e. $H_{1}=0$, then

$$
\left[\nabla_{i} \nabla_{j} F\right]=H_{2} N_{i} N_{j}+\left(N_{j} Z_{i}^{\alpha}+N_{i} Z_{j}^{\alpha}\right) B_{\alpha}^{\beta} \nabla_{\beta} H_{0}+Z_{i}^{\alpha} Z_{j}^{\beta} \nabla_{\alpha} \nabla_{\beta} H_{0} .
$$

Finally, if $F$ is continuous along with its derivatives, i.e. $H_{0}=H_{1}=0$, then the compatibility conditions have the particularly simple form

$$
\left[\nabla_{i} \nabla_{j} F\right]=H_{2} N_{i} N_{j} \text {. }
$$


Let us also use the main compatibility condition (43) to derive the general expression for the jump discontinuity in the Laplacian $\nabla_{i} \nabla^{i} F$. Raising the index $j$ and contracting with $i$, we find

$$
\begin{aligned}
{\left[\nabla_{i} \nabla^{i} F\right]=} & H_{2} N_{i} N^{i}+\left(N_{i} Z^{i \alpha}+N^{i} Z_{i}^{\alpha}\right) \nabla_{\alpha} H_{1}-B_{i}^{i} H_{1} \\
& +\left(N_{i} Z^{i \alpha}+N^{i} Z_{i}^{\alpha}\right) B_{\alpha}^{\beta} \nabla_{\beta} H_{0}+Z_{i}^{\alpha} Z_{\beta}^{i} \nabla_{\alpha} \nabla^{\beta} H_{0} .
\end{aligned}
$$

Recall that $N_{i} N^{i}=0, N_{i} Z^{i \alpha}=N^{i} Z_{i}^{\alpha}=0$, and $Z_{i}^{\alpha} Z_{\beta}^{i}=\delta_{\beta}^{\alpha}$. Therefore,

$$
\left[\nabla_{i} \nabla^{i} F\right]=H_{2}-B_{i}^{i} H_{1}+\nabla_{\alpha} \nabla^{\alpha} H_{0} .
$$

Meanwhile, for the invariant $B_{i}^{i}$, we have

$$
B_{i}^{i}=B_{\alpha \beta} Z^{i \alpha} Z_{i}^{\beta}=B_{\alpha \beta} S^{\alpha \beta}=B_{\alpha}^{\alpha},
$$

where the invariant $B_{\alpha}^{\alpha}$ is known as the mean curvature. Thus, finally, we have

$$
\left[\nabla_{i} \nabla^{i} F\right]=H_{2}-B_{\alpha}^{\alpha} H_{1}+\nabla_{\alpha} \nabla^{\alpha} H_{0} .
$$

This completes the main technical challenge of our investigation, that is, to derive the general compatibility conditions. In order to apply the obtained formulas to specific situations, we must simply determine the values of the surface fields $H_{0}, H_{1}$, and $H_{2}$. For the classical Newtonian potential, as well as the potentials associated with simple and double layers, these fields can be determined by analyzing the well-established partial differential equations and boundary conditions that govern them. This task will be accomplished in the next three sections.

\section{The Classical Newtonian Potential and Weingarten's \\ Condition}

Recall that the Newtonian potential $V$ is defined by the integral equation

$$
V_{\rho}(Z)=-\int_{\Omega^{+}} \frac{\rho(Z)}{\left|\vec{Z}(Z)-\vec{Z}\left(Z^{*}\right)\right|} d Z^{*}
$$

where $\Omega^{+}$is the domain occupied by the mass distribution $\rho(Z)$, as illustrated in Figure 2.

The potential $V$ satisfies the Poisson equation

$$
\nabla_{i} \nabla^{i} V=-4 \pi \rho
$$

inside $\Omega^{+}$and the Laplace equation

$$
\nabla_{i} \nabla^{i} V=0
$$

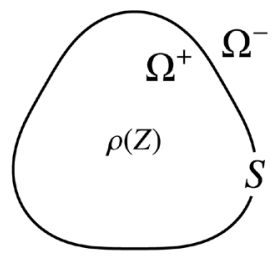

Figure 2. A self-gravitating body $\Omega^{+}$with density $\rho(Z)$ and boundary $S$. 
inside $\Omega^{-}$. Furthermore, $V$ is continuous across $S$ along with its derivatives, i.e.

$$
[V]=0
$$

and

$$
N^{i}\left[\nabla_{i} V\right]=0
$$

In other words,

$$
H_{0}=H_{1}=0 \text {. }
$$

Thus, from equation

$$
\left[\nabla_{i} \nabla^{i} F\right]=H_{2}-B_{\alpha}^{\alpha} H_{1}+\nabla_{\alpha} \nabla^{\alpha} H_{0}
$$

we observe that

$$
\left[\nabla_{i} \nabla^{i} F\right]=H_{2}
$$

As a result, Equations (51), (52) tell us that

$$
H_{2}=-4 \pi \rho \text {. }
$$

Having determined $H_{0}, H_{1}$, and $H_{2}$, we can conclude from Equation (46), that

$$
\left[\nabla_{i} \nabla_{j} V\right]=-4 \pi \rho N_{i} N_{j}
$$

which is precisely Weingarten's condition announced in [1].

\section{Simple Layers}

Recall that potential $V_{\sigma}$ induced by the simple layer distribution $\sigma$ on the surface $S$ is defined by Equation (2). Similarly to the Newtonian potential $V, V_{\sigma}$ is twice differentiable away from $S$ where it satisfies the Laplace equation

$$
\nabla^{i} \nabla_{i} V_{\sigma}=0
$$

On the other hand, the continuity conditions across the interface $S$ are distinct from those of the Newtonian potential $V$. Specifically, while $V_{\sigma}$ is continuous across $S$, i.e.

$$
\left[V_{\sigma}\right]=0
$$

its first derivative experiences a jump discontinuity captured by the equation

$$
N^{i}\left[\nabla_{i} V_{\sigma}\right]=-4 \pi \mu \text {. }
$$

Thus,

$$
H_{0}=0
$$

and

$$
H_{1}=-4 \pi \mu \text {. }
$$

Next, from equation

$$
\left[\nabla_{i} \nabla^{i} F\right]=H_{2}-B_{\alpha}^{\alpha} H_{1}+\nabla_{\alpha} \nabla^{\alpha} H_{0}
$$

it follows that 


$$
H_{2}=B_{\alpha}^{\alpha} H_{1}+\left[\nabla_{i} \nabla^{i} F\right] .
$$

Since from Equation (59), it is apparent that

$$
\left[\nabla_{i} \nabla^{i} F\right]=0 \text {, }
$$

we conclude that

$$
H_{2}=-4 \pi \mu B_{\alpha}^{\alpha} .
$$

Once again, having determined $H_{0}, H_{1}$, and $H_{2}$, we can use the compatibility conditions (44) to arrive at the equation

$$
\left[\nabla_{i} \nabla_{j} V_{\sigma}\right]=-4 \pi\left(\sigma B_{\alpha}^{\alpha} N_{i} N_{j}+\left(N_{i} Z_{j}^{\alpha}+N_{j} Z_{i}^{\alpha}\right) \nabla_{\alpha} \sigma-\sigma B_{i j}\right) .
$$

This is our main result concerning the simple layer potential $V_{\sigma}$.

In conclusion, we note that for the special case of a flat interface $S$, characterized by $B_{\alpha \beta}=0$ and therefore $B_{\alpha}^{a}=0$ and $B_{i j}=0$, we have

$$
\left[\nabla_{i} \nabla_{j} V_{\sigma}\right]_{-}^{+}=-4 \pi\left(N_{i} Z_{j}^{\alpha}+N_{j} Z_{i}^{\alpha}\right) \nabla_{\alpha} \sigma \text {. }
$$

\section{Double Layers}

Recall that the double layer potential $V_{\delta}$ is defined by the integral Equation (3). Similar to $V_{\sigma}$, the potential $V_{\delta}$ is also twice differentiable away from the interface $S$ where Laplace equation

$$
\nabla^{i} \nabla_{i} V_{\delta}=0
$$

away from the boundary $S$. However, at the boundary $S, V_{\delta}$ is discontinuous. Its jump discontinuity is described by the equation

$$
\left[V_{\delta}\right]=-4 \pi \tau \text {. }
$$

Its normal derivatives, on the other hand, are continuous, i.e.

$$
N^{i}\left[\nabla_{i} V_{\delta}\right]=0 .
$$

In other words

$$
H_{0}=-4 \pi \tau
$$

and

$$
H_{1}=0 .
$$

In order to determine $\mathrm{H}_{2}$, let us once again turn our attention to equation

$$
\left[\nabla_{i} \nabla^{i} F\right]=H_{2}-B_{\alpha}^{\alpha} H_{1}+\nabla_{\alpha} \nabla^{\alpha} H_{0}
$$

from which we observe that

$$
\left[\nabla^{i} \nabla_{i} V_{\delta}\right]=H_{2}-4 \pi \nabla_{\alpha} \nabla^{\alpha} \tau .
$$

Since, according to Equation (69), the Laplacian is zero on either side of the boundary, $\left[\nabla^{i} \nabla_{i} V_{\delta}\right]=0$ and therefore we find that

$$
H_{2}=4 \pi \nabla_{\alpha} \nabla^{\alpha} \tau
$$

Thus, from Equation (43), we arrive at the compatibility condition 


$$
\left[\nabla_{i} \nabla_{j} V_{\delta}\right]=4 \pi\left(N_{i} N_{j} \nabla_{\alpha} \nabla^{\alpha} \tau-\left(N_{i} Z_{j \alpha}+N_{j} Z_{i \alpha}\right) B^{\alpha \beta} \nabla_{\beta} \tau-Z_{i}^{\alpha} Z_{j}^{\beta} \nabla_{\alpha} \nabla_{\beta} \tau\right)
$$

This is our main result concerning the double layer potential $V_{\delta}$.

As before, note the special case of a flat interface, i.e.

$$
\left[\nabla_{i} \nabla_{j} V_{\delta}\right]=4 \pi\left(N_{i} N_{j} \nabla_{\alpha} \nabla^{\alpha} \tau-Z_{i}^{\alpha} Z_{j}^{\beta} \nabla_{\alpha} \nabla_{\beta} \tau\right)
$$

\section{Conclusion}

The three most popular distributions in the classical potential theory are the volumetric distribution, described by the singular three-dimensional Integral (1), the simple layer, described by the two-dimensional singular surface Integral (2), and the double layer, described by the two-dimensional singular Integral (3). The second derivatives of the resulting potential experience finite jump discontinuities at the corresponding interfaces. For the volumetric distribution, the second-derivative discontinuity is given by Equation (58) established by Weingarten [1] in 1887. In this paper, we provided the analogous relationships (67), (76) the simple layer and double layers.

\section{Conflicts of Interest}

The authors declare no conflicts of interest regarding the publication of this paper.

\section{References}

[1] Weingarten, J. (1887) Zur Theorie der Flachenpotentials. Acta Mathematica, 10, 303-309.

[2] Vladimirov, V.S. (1971) Equations of Mathematical Physics. Dekker, New York.

[3] Kellogg O. (2010) Foundations of Potential Theory. Dover Publications, New York.

[4] Courant, C. and Hilbert, D. (2008) Methods of Mathematical Physics: Partial Differential Equations. Wiley, Hoboken.

[5] Korn, G.A., and Korn, T.M. (1968) Mathematical Handbook for Scientists and Engineers. McGrow-Hill, New York.

[6] Sretensky, L.N. (1946) Theory of the Newtonian potential. State Technical and Theoretical Publishing House, Leningrad.

[7] Smirnov, V.I. (1964) A Course of Higher Mathematics. Pergamon Press, Oxford.

[8] McConnell, A.J. (1957) Applications of Tensor Analysis. Dover Publications, Dover.

[9] Thomas, T.Y. (1961) Plastic Flow and Fracture in Solids. Elsevier, Amsterdam.

[10] Grinfeld, M. (1991) Thermodynamic Methods in the Theory of Heterogeneous Systems. Longman, Harlow.

[11] Grinfeld, P. (2014) Introduction to Tensor Analysis and the Calculus of Moving Surfaces. Springer, New York. 